\title{
FLUID-STRUCTURE INTERACTION MODELING: FIRST APPLICATIONS AT CNES
}

\section{J. Herpe and S. Petitot}

Space Transport Systems CNES Launcher Directorate

Evry, France

This paper deals with the numerical methodologies used at CNES DLA to set up Fluid-Structure Interaction (FSI) calculations. The numerical strategy developed is based on the code coupling which allows the communication between two specific codes. In fact, to model launcher's components, CNES uses various specialized codes (for thermal, mechanical, fluids, combustion applications, etc.), to seek individual solutions of the highly complex problem. Then, a third code allowing communication between computational fluid dynamics (CFD) and finite element analysis (FEA) (interpolations between different meshes) and exchanging shared variables (mainly, pressures and displacements) is required for FSI problems. One of the major application fields identified concerns the solid propulsion. Two problems are investigated to perform coupled calculations with increasing complexity. The first one is about the Solid Rocket Motor (SRM) ignition. This transient phase can be decomposed by successive steady-states with step-by-step increasing of internal motor pressure. This allows performing unidirectional coupling with both a script developed at CNES and the MpCCI software. The second one concerns thrust oscillations of SRM. A bidirectional approach is used to simulate the behavior of a flexible obstacle, made of elastomer, protruding in a cold gas flow of an experimental test bench developed to reproduce oscillating pressure phenomena at a reduced scale.

\section{INTRODUCTION}

Launcher design, development activities, and postflight analysis often help pointing out problems that involve strong coupling between different physical behavior. Several examples can be found among the three main domains of the propulsion:

\footnotetext{
This is an Open Access article distributed under the terms of the Creative Commons Attribution-Noncommercial License 3.0, which permits unrestricted use, distribution, and reproduction in any noncommercial medium, provided the original work is properly cited.
} 
(1) liquid propulsion: aeroelastic coupling between choc oscillations appearing inside the expanded nozzle and its wall deformations, aerothermal solicitations leading to crack formations on injectors in cryogenic combustion chamber, aerothermomechanical coupling involved on "side loads" phenomena with deformation of the cooling ducts along Vulcain 2 nozzle;

(2) cryogenics tanks: thermomechanical coupling for Internal Thermal Protection design, hydroelastic and sloshing vibrations which occur in stability studies ("pogo" effect, attitude control of launcher); and

(3) solid propulsion: aeromechanics coupling for modeling "Front Thermal Protection" (PTF) behavior (bending and vibrations) impacting Pressure Oscillation phenomena, aeromechanical coupling necessary for solid propellant mechanical diagnostic (initiation of cracks) during motor ignition (pressurization).

Among these three domains, the solid propulsion is of particular interest for the present authors. In fact, for historical reasons, the pressure oscillations appearing in Ariane $5 \mathrm{SRMs}$ is a fundamental research subject at CNES/DLA, especially for a better understanding of the physical phenomena leading to these oscillations. Moreover, from a numerical point of view, the solid applications allow improvements in the simulation strategy due to the kind of the phenomena encountered in solid propulsion.

To perform fluid and solid simulations, specific codes are usually employed at CNES. Then, in order to model the fluid--structure interaction, a third code allowing communication between $\mathrm{CFD}$ and FEA is required to exchange shared variables (mainly, pressure and displacement values). In this paper, Fluent [1] is used for fluid simulation and Abaqus [2] for the structural analysis. Two methods are studied for coupling aspects. The first is based on a script developed at CNES. The second is based on the MpCCI [3] software that already includes adapters for the commercial codes Fluent and Abaqus.

In a general manner, when fluid-structure interaction phenomena occur, the flow pressure bends the structure that can modify, in exchange, the flow field. According to the relevance of the impact of the structural bending on the flow pressure, it is possible to consider or ignore in the numerical exchange scheme (Fig. 1). In the case of a one-way coupling scheme, the exchange is only done from the fluid to the solid (unidirectional coupling). In a two-way coupling, the exchange is done in both directions (bidirectional coupling).

Then, this paper focuses on two pure numerical cases. The first is related to a motor ignition problem. The influence of the structure on the fluid flow is neglected and a one way coupling approach is used. A specific script of exchange values developed at CNES is done. It demonstrates the feasibility and points out the main difficulties. Then, a comparison is made with calculations via MpCCI. The second case is considering a two-way coupling FSI problem. This numerical 


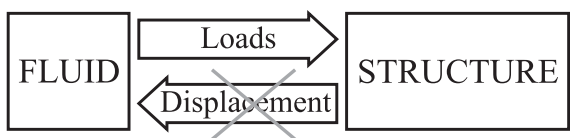

(a)

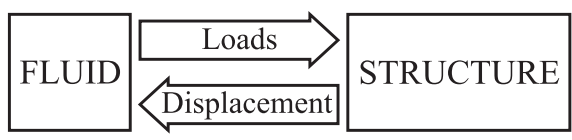

(b)

Figure 1 One-way (a) and two-way (b) coupling schemes

case is based on the MICAT bench test from University of Poitiers, France, that allows investigating the coupling between a flexible obstacle (elastomer) movement and a fluid flow.

\section{ONE-WAY COUPLING APPLICATION}

The first numerical application is related to the study of the ignition phase (phase II in Fig. 2b), or pressurization of a solid rocket motor (Figs. $2 a$ and $2 b$ ). The very fast increase of pressure in the motor induces stresses on the propellant block(s) and strain of the burning surfaces, modifying boundary limits for the fluid flow. The regression of the propellant block due to combustion is not taken into account. In fact, the characteristic time of phase II is very short compared

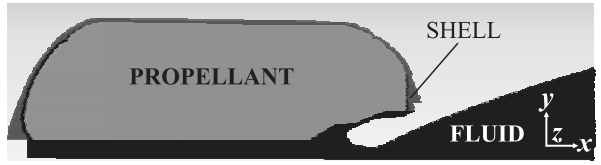

(a)

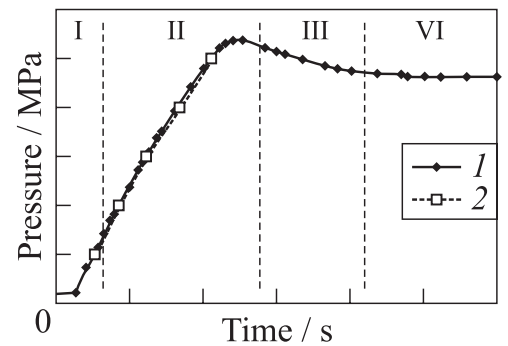

(b)

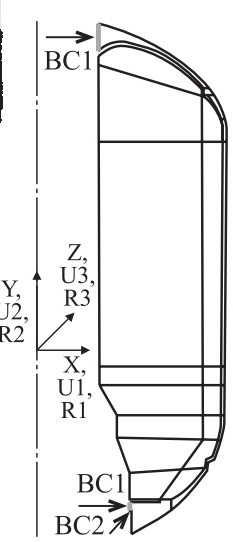

\begin{tabular}{|c|l|l|l|l|l|l|}
\hline & U1 & U2 & U3 & R1 & R2 & R3 \\
\hline $\begin{array}{c}\text { BC1 } \\
\text { blocked } \\
\text { DoF }\end{array}$ & $\mathrm{X}$ & & & & $\mathrm{X}$ & $\mathrm{X}$ \\
\hline $\begin{array}{c}\text { BC2 } \\
\text { blocked } \\
\text { DoF }\end{array}$ & & $\mathrm{X}$ & $\mathrm{X}$ & $\mathrm{X}$ & & \\
\hline
\end{tabular}

$\mathrm{BC} 2$

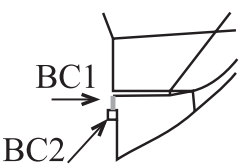

(c)

Figure 2 Solid rocket motor configuration $(a)$; pressure evolution in the SRM $(b)$; and boundary condition for the structural analysis $(c)$ (BC1 - axial symmetric condition and $\mathrm{BC} 2$ - block condition) 

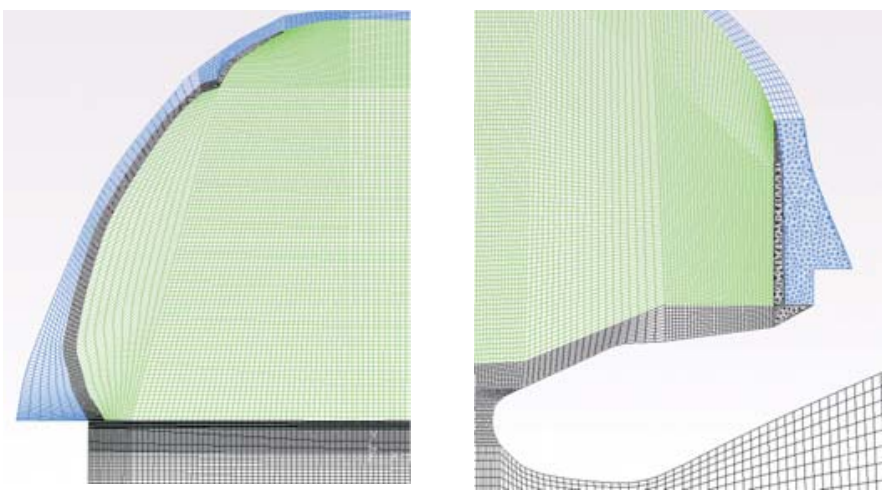

(a)

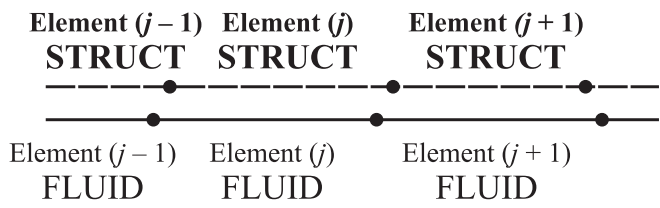

(b)
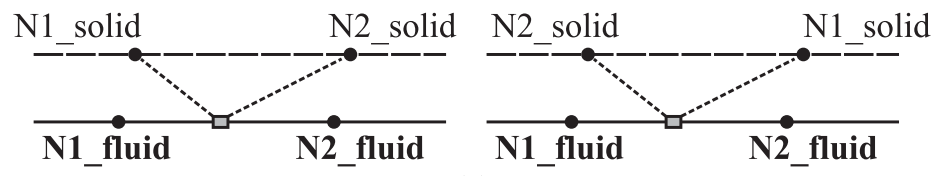

(c)

Figure 3 Mesh details at the top and the bottom of the SRM (a); scheme of discrepancies due to the round-off error when importing the Gmsh mesh in Fluent and Abaqus (b); and Central Point Distance Evaluation procedure (c). (Refer Herpe and Petitot, p. 730.)

with the characteristic time of burning surface regression. Thus, this study focuses on the strain due to the pressure loads.

A simplified two-dimensional (2D) axisymmetric model is considered. The fluid and solid meshes which are fitted at the fluid/structure interface (Fig. 3) are generated in the Gmsh software [4] and are exported into the corresponding software.

Hybrid elements are used to model propellant behavior as it is fully incompressible, while incompatible mode elements are used to mesh the shell part. The mechanical behavior of propellant and shell are based on the hypothesis of a linear elastic law. The boundary conditions imposed on solid are plotted in Fig. $2 c$. 
The fluid flow is obtained using a pressure-based implicit solver with pressurevelocity coupled algorithm. The second-order schemes are used for pressure, density, velocity, and temperature discretization algorithms. The boundary conditions are obtained using the pressure curve of Fig. $2 b$ and the classical internal ballistic laws of the SRM. A mass flow inlet is imposed at the fluid/propellant interface. At the outlet of the nozzle, the pressure is fixed. The other fluid boundary conditions are supposed to be adiabatic walls. It is supposed that the flow is a nonreactive monophasic ideal gas flow. The viscosity, thermal conductivity, and specific heat at constant pressure are supposed to be constant.

As a first approximation, a "step-by-step" approach is used to model the pressurization of the SRM. For each of the five operating points (squares in Fig. 2b), a one-way cosimulation is used. From the pressure value of the operating point and with the classical ballistic laws of the SRM, the flow boundary conditions are obtained. A steady-state simulation is performed with Fluent. The surface forces obtained are only transfered once from the fluid model to the solid model (Abaqus) where they are applied as boundary conditions to compute the stress distribution. At the following operating point, the fluid model takes into account the deforming surface of the propellant block and the new boundary conditions are calculated with the internal ballistic laws. So, the pressure load obtained is sent to the mechanical model. This procedure is implemented until the fifth step.

Two methodologies for the data exchange, from the fluid model to the solid model, are explained in the next subsection. The first is based on the script developed at CNES, while the second is based on the MpCCI software.

\subsection{Script Methodology}

For each point corresponding to a given motor pressure (see Fig. 2b), the script procedure is as follows:

1. Boundary conditions: for a given operating pressure, the inlet mass flux boundary conditions are calculated based on the classical internal ballistic law.

2. Fluid simulation: a steady-state calculation for the fluid flow is performed using Fluent.

3. Exchange procedures: the pressure distribution along propellant surface(s) is read with a specific Fluent User Defined Function (UDF) developed for this purpose. The specific Python script is used to match the CFD pressure values with finite elements and to generate the input file for structural analysis. 
4. Solid simulation: the finite-element structural analysis is made for the deformed configuration of the propellant block(s) and the new geometry is updated.

5. Remeshing procedure: the fluid domain is manually remeshed with the "deformed" boundary limits.

6. Step 1 is made again with the subsequent internal pressure value.

The exchange procedure (step 3) is strongly linked to the mesh. If the fluid and propellant meshes are not conformal, it is necessary to apply interpolation algorithms to exchange the values. As a first simplified approach, it has been chosen to use a conformal mesh at the fluid/structure interface (Fig. $3 a$ ) to ensure exact matching. However, even though the fluid and the propellant meshes have been both generated in Gmsh, the export operation in Fluent and Abaqus leads to some discrepancies (Fig. $3 b$ ), probably due to a round-off error. This was a reason for developing a script with a central point distance evaluation algorithm (Fig. 3c). The principle is based on the calculation of the distances between the central points of fluid face elements and the nodes of structural elements and to find the nearest structural element for a given fluid element (Fluent UDF lists also the fluid node coordinates while recording pressure values). It is not influenced by node configuration and does not need reconstruction of mesh information ("low" computer memory cost). This algorithm can also be used for a three-dimensional (3D) mesh, with the evaluation of three (triangular element at the surface) or four (hexahedral) distances.

\subsection{MpCCI Methodology}

The same calculation is performed with the MpCCI code ensuring coupling between CFD and FEA. Data exchanges are then directly performed between the two conformal meshes by MpCCI, without customer intervention. The "step-bystep" process is slightly different from that used with the script method due to the specific features the "MpCCI" coupling procedure. The main differences are in step 3 that include steps $3-5$ of the previous script methodology. This is done thanks to the MpCCI automatic procedure.

1. Boundary conditions: for the given functioning motor pressure, internal ballistics is evaluated. Inlet mass flux boundary conditions are calculated.

2. Fluid simulation: a steady calculation for the fluid flow is performed using Fluent in order to evaluate the pressure distribution along propellant $\operatorname{surface}(\mathrm{s})$. 
3. Exchange procedure, finite-element simulation and remeshing: after starting the MpCCI server, the exchange is interactively done with the graphical user interface (GUI) of Fluent. The solution is first initialized with the corresponding button in the "on demand init and exit" portion of the MpCCI control panel. The pressure is sent to Abaqus, and the node position is sent to Fluent by clicking on the "exchange" button at the panel. With the pressure load sent by Fluent UDF, the finite-element simulation can be done and node displacement can be calculated and sent to Fluent. The "steady update mesh" button allows updating the Fluent mesh. Eventually, the "Finalize" button is used to finish the simulation.

4. The new fluid mesh can be used for the next step.

At step $i+1$, it is necessary for the structural analysis to take into account the solution, obtained at step $i$ (not only the propellant node displacement but also the fields of strain and stress). In order to do it and due to the fact that the MpCCI procedure is not able to take into account the multistep procedure of Abaqus, the restart procedure of Abaqus is used.

\subsection{Results}

Comparison between both coupling methodologies is based on both Von Mises Stress values and displacements obtained respectively at probe elements (central points) and probe nodes in the shell and propellant grain.

For displacement, a qualitative analysis can only be made as nodal displacements are small compared to the motor size. As shown in Fig. 4, the final position of the propellant grain surface in the rear part of the motor are quite similar for the two methodologies.

Also, the analysis of the Von Mises Stress field (Fig. 5) shows that the re-

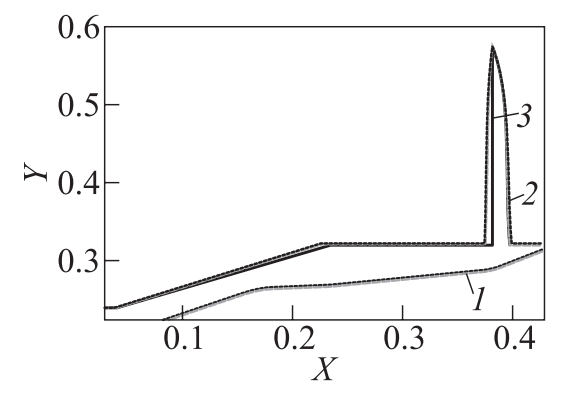

Figure 4 End surface position for both MpCCI (1) and Script (2) coupled calculations, zoom in the rear part of the motor where displacements are most significant; 3 - undeformed sults from script and MpCCI methodology are comparable: good agreement between the two methodologies is also found considering Von Mises Stress Evolution with pressure increase (step) for points located inside the propellant, near the interface.

Thus, this first simplified "step-by-step" approach using MpCCI was validated by comparing with Script results. Further works have to investigate a bidirectional coupling using unsteady calculations with MpCCI coupling only. 
S. Mises (Avg: 75\%)

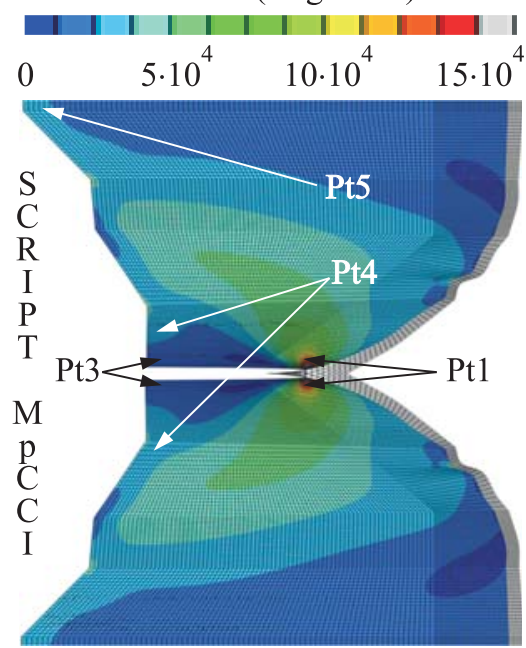

S. Mises (Avg: 75\%)
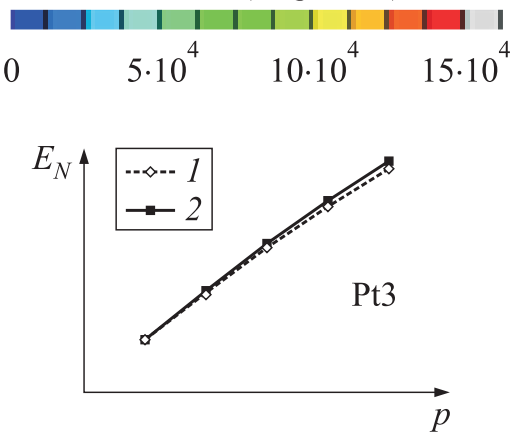
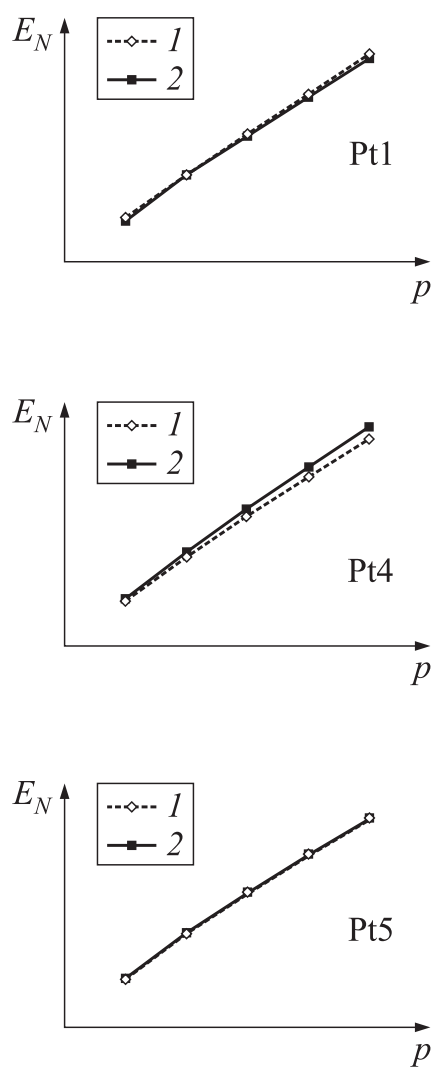

Figure 5 Comparison of Von Mises Stress field at rear part of the motor (above) and Von Mises Stress evolution with pressure rising (step) within some propellant located points: 1 - MpCCI; and 2 - Script. (Refer Herpe and Petitot, p. 734.)

\section{TWO-WAY COUPLING}

\subsection{Description of the Problem}

The solid rocket motors of Ariane 5 are made of three propellant blocks separated by thermal protections (PTS2 and PTS3). During the internal combustion, the fire front progresses and the thickness of the propellant blocks decreases. As a consequence, the thermal protection PTS3 protrudes and disturbs the gas flow, producing one of the major effects driving the pressure oscillations (Fig. 6). In 

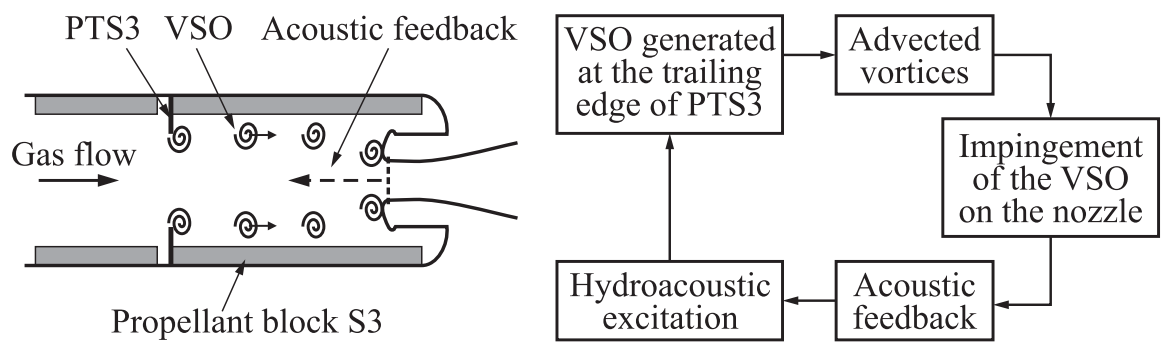

Figure 6 Pressure oscillations loop

fact, the vortex shedding from obstacle (VSO) is generated at the trailing edge of PTS3 and impinges on the rocket motor nozzle. This interaction between the vortex and the nozzle is responsible for an acoustic feedback that excites the following detached VSOs.

Cold-flow experiments [5-8] and numerical simulations [9-11] have been dedicated to study the parameters affecting pressure oscillations. Among them, the bending of the thermal protection PTS3, which is made of a flexible inhibitor, has been identified. However, the quantification of thermal protection bending by means of numerical simulation is still challenging despite the improvements of numerical analyses and computing power. In order to evaluate the capability of the approach based on the Fluent/MpCCI/Abaqus coupling codes simulating this FSI phenomenon, this paper is focused on the MICAT configuration test rig described in the following subsection.

\subsection{Vortex Shedding Obstacle Study: Cold Flow Test Bench MICAT}

The MICAT configuration (Fig. 7) is an experimental cold air test bench developed to reproduce oscillating pressure phenomena at a reduced scale. The propellant block combustion is simulated by a parietal gas injection through a porous material. The rig is constituted by three propellant blocks, two thermal protections (sheet of a specific material), and a nozzle. The rig is adjustable in order to define different operating points corresponding to the different combustion times of a solid rocket motor.

An operating point, near the end of combustion, also called resonant configuration, is numerically investigated afterwards (Fig. 8). At this operating point, the propellant block S1 has completely burnt. Only combustion of propellant blocks S2 and S3 is taken into account. All other surfaces of the rig are represented by the adiabatic walls. The PTS2 is supposed fixed and normal to the axial flow direction. Due to its material characteristics, the PTS3 could bend under the influence of gas flow pressure. 


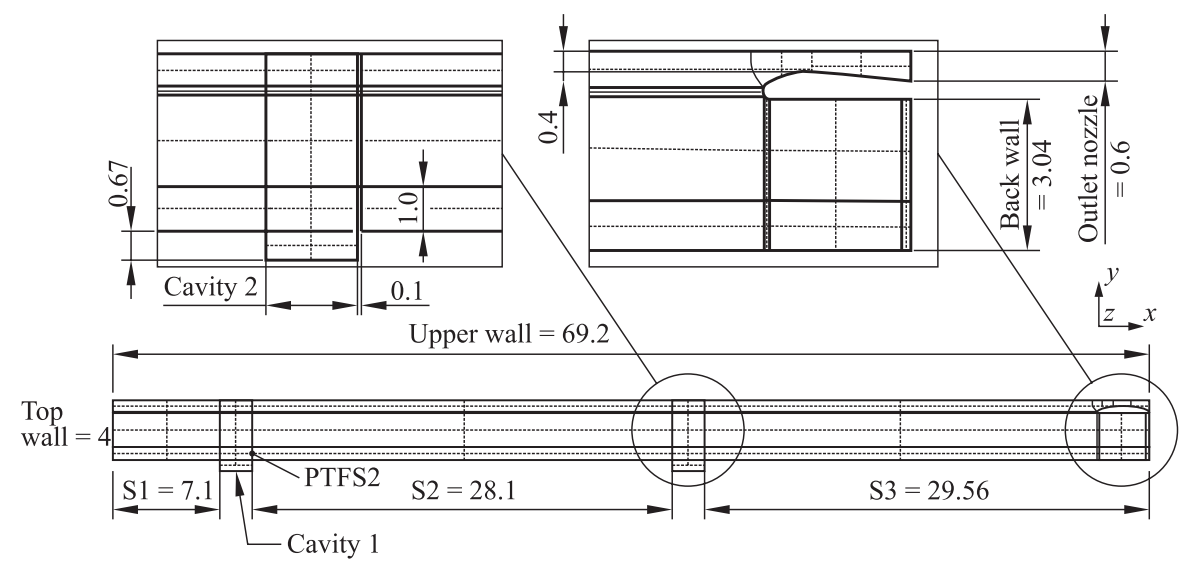

Figure 7 Cold flow MICAT test bench, dimensions are in millimeters

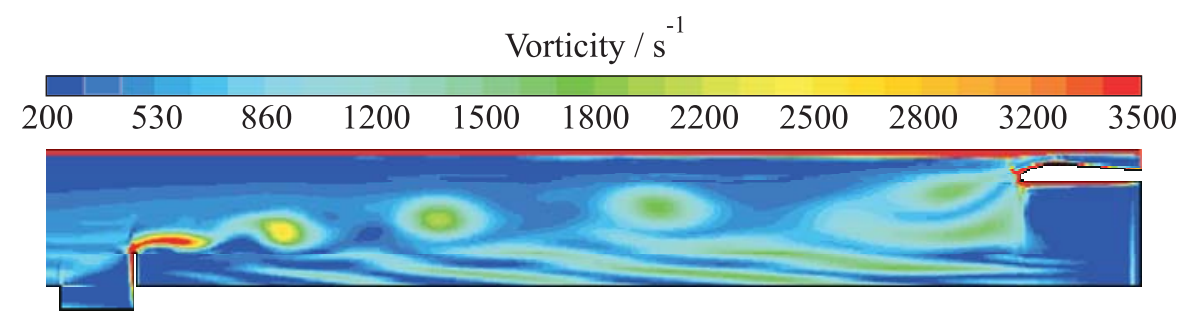

Figure 8 Contour of vorticity downstream the PTS3. (Refer Herpe and Petitot, p. 736.)

The acoustic response of the MICAT cavity is analyzed with a pressure sensor mounted at the top wall (see Fig. 7).

\subsection{Numerical Fluid-Structure Interaction Models}

The fully coupled physical phenomena, summarized in subsection 3.2, were simulated using the bidirectional approach available in the MpCCI software [3]. This cosimulation software linked the Fluent CFD code [1] (gas flow model) and the Abaqus Finite Elements code [2] (thermal protection PTS3 model). The models and the schemes used in each code are described below.

\subsubsection{Gas flow model}

A 2D numerical model was used. The gas flow was a single-phase, compressible (ideal gas), and laminar. The combustion reactions were not taken into account. The viscosity, thermal conductivity, and specific heat at constant pressure were supposed to be constant. 
The boundary conditions were as follows:

- at the adiabatic walls, the flux was set zero and the no-slip shear condition was imposed;

- the mass flux was fixed at the injection side; and

- the static pressure was fixed at the nozzle outlet (it was taken into account when the flow was subsonic. After nozzle choking, the pressure was extrapolated from the inlet motor condition).

The density-based solver was used with a Roe scheme (third-order MUSCL (Monotone Upwind Scheme for Conservation Laws) discretization scheme). A Runge-Kutta explicit time scheme was used. The Courant-Friedrich-Levy (CFL) number was equal to 1.6. The Green Gauss gradient cell-based option was used.

Two of the dynamic mesh methods available in Fluent were used in the FSI model. The first was used for vibrating phenomena and the second for high strain. The first called "the smoothing method" was based on the Hook's Law. The edges between any two mesh nodes were simulated as a network of interconnected springs characterized by their stiffness. A displacement at a given node generated a force proportional to the displacement along all the springs connected to the node. This approach was useful for small deforming meshes. However, at high deformations, it was necessary to use a remeshing method. The strategy of this second method consisted in packing together the cells if the specific criteria defined by the user (cell skewness, etc.) were not respected. This approach only worked with triangular meshes which limited the mesh choice (Fig. 9b). This mesh constraint will be analyzed by comparing the triangular mesh solution with the quadrangle mesh solution (Fig. 9a) for the case of a straight thermal protection.

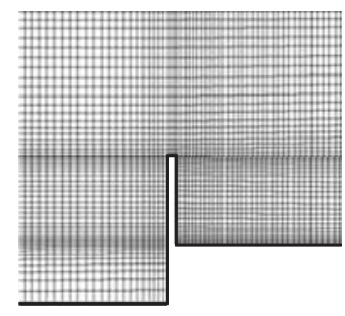

(a)

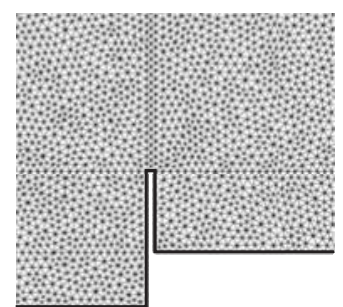

(b)

Figure 9 Gas flow meshes: (a) quadrangle mesh; and (b) triangular mesh 


\subsubsection{Structural model}

The thermal protection PTS3 was made of an arbitrary incompressible material (the Poisson coefficient is equal to 0.49). The mechanical behavior was based on the hypothesis of a linear elastic law.

The boundary conditions for the PTS3 were as follows: the foot of the PTS3 was fixed $\left(U_{1}=U_{2}=U_{3}=U R_{1}\right.$ $\left.=U R_{2}=U R_{3}=0\right)$. The implicit solver of Abaqus was used.

The mesh was made of the hybrid-reduced elements CPE4RH. When the structural mesh was fitted to the triangular fluid mesh (Fig. 10a), Hourglass problems could appear. Therefore, the mesh defined in Fig. $10 b$ was used.

The behavior of thermal protection was investigated at the black tip node of Fig. 10.

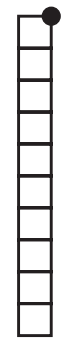

(a)

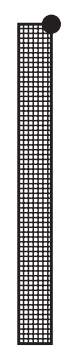

(b)

Figure 10 The PTS3 meshes

\subsubsection{Coupling model in MpCCI}

As explained in previous subsections, an ex-

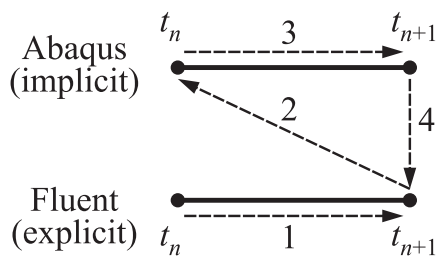

Figure 11 Serial scheme plicit scheme was used for the fluid model whereas for the structural model, an implicit scheme was applied. In such a case, it is recommended in [2] to use a serial coupling scheme with the code using the explicit time integration being the leader code. Thus, the code using explicit time integration computes the solution at $t_{n+1}$ based on the solution at $t_{n}$ and passes the forcing quantity to the implicit solver, which computes the solution at $t_{n+1}$ (Fig. 11).

In the fluid model, the time step was not constant due to the explicit scheme used (CFL criterion was imposed). Therefore, a coupling time step to define the rendezvous between Abaqus and Fluent is not constant. Nevertheless, the rendezvousing scheme could be defined in MpCCI specifying a variable coupling step size. In this case, it was necessary to choose between Fluent or Abaqus to send or receive the time increment size. Thus, it was supposed that the sender was Fluent. Moreover, it enforced the exact target time using the subcycling approach in Abaqus. The initial quantities are transferred by Fluent, and then exchanged and received by Abaqus.

The values exchanged between the CFD code and the Finite-Elements code during this process are, respectively, the pressure (overpressure) and the node 
position $\left(N_{\text {position }}\right)$.

As the mechanical and the fluid meshes are not fitted, the exchange values are obtained by mesh interpolation using the minimal distance algorithm.

\subsection{Flow Model Validation}

The validation of the Fluent model was based on a numerical comparison with the CFD code CPS_P [12] dedicated to the solid rocket motor applications.

In this validation case, the thermal protection PTS3 was not deformable and remained normal to the axial flow direction. The CPS_P model was the same as that in Fluent. Both of the previous meshes were studied (see Fig. 9). The nozzle was choked the pressure signal obtained in the sensor was established as periodic (Fig. 12). The simulation was stopped when a sufficient number of periods was obtained to make a precise Fast Fourier Transform (FFT).

The results show a good agreement between CPS_P and Fluent in the case of the quadrangular mesh. The frequencies of acoustic modes were the same for the triangular and the quadrangular meshes and also for CPS_P and Fluent. There
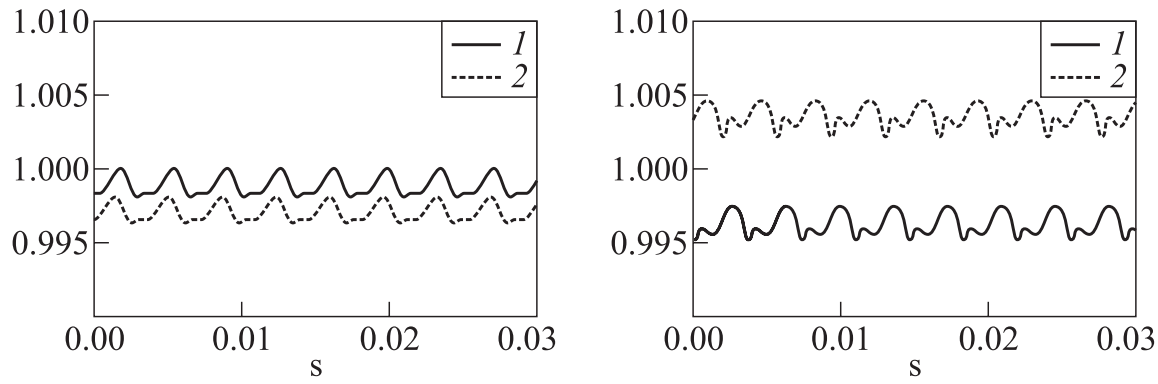

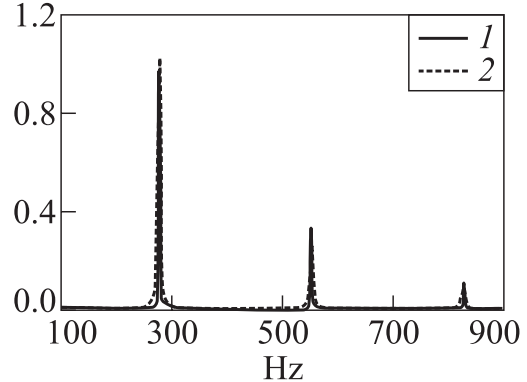

(a)

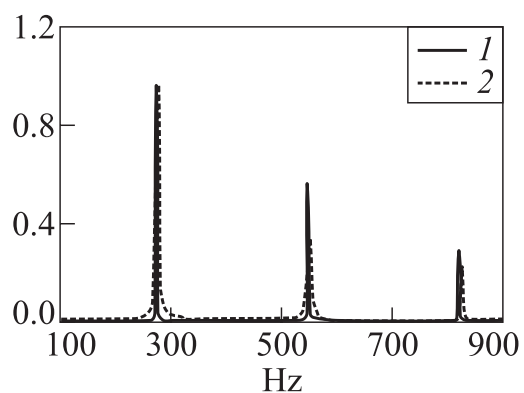

(b)

Figure 12 Fast Fourier Transform of the pressure signal for the quadrangle $(a)$ and triangle (b) mesh: 1 - CPS; and 2 - Fluent 
were some differences in the amplitude between quadrangular and triangular meshes for both CFD codes. These differences were focused on the second and the third acoustic modes. The main difference between both CFD codes was observed for the second mode. As a first approximation, it was supposed that the Fluent model and the triangular mesh used were good enough. In fact, the pressure signal and the fundamental acoustic mode of the FFT were nearly the same for both meshes and both codes.

\subsection{Cosimulation Analysis}

Two strategies could be used for the cosimulation analysis. The first consisted in using the previous gas flow solution (subsection 3.4) and direct running of unsteady cosimulation. The second consisted in running a static cosimulation to obtain the first bending of the PTS3 and then running an unsteady simulation from a converged gas flow solution. Due to the relatively short fluid time step, the static stage was introduced to save CPU time.

For the static stage, the steady solver of Fluent and the static solver of Abaqus were used. In MpCCI, the exchange was manually done with the MpCCI control

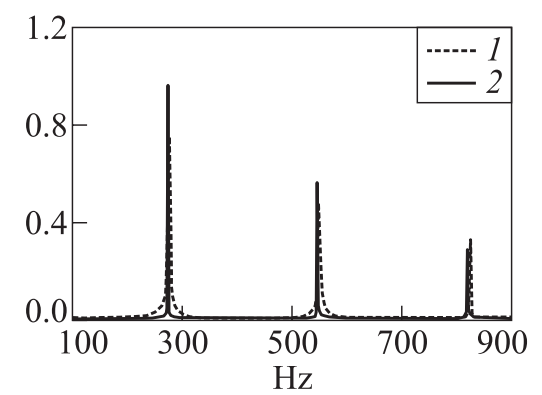

Figure 13 Fast Fourier transform of the pressure signal for the bending PTS3 (1) and the straight PTS3 (2)

panel of the Fluent software, and no rendezvousing scheme was used. The quantities were still initially exchanged by Fluent and received by Abaqus. After this step, the unsteady cosimulation could be started by loading the corresponding Fluent case and using the restart procedure of Abaqus.

A middle analysis at the end of the steady cosimulation, confirmed the influence of the bending on the pressure signal (Fig. 13). The first-mode amplitude of the straight PTS3 is $20 \%$ higher than the amplitude of the bending PTS3.

The results of unsteady cosimulation show (Fig. 14) that the PTS3 oscillates around an average position. The oscillations of the PTS3 decrease with time leading to small vibrations. After the numerical transition period, the pressure signal (Fig. 15) is seen to stabilize.

In order to learn whether the PTS3 oscillation has an impact on the pressure signal, the cosimulation was stopped and switched to a solely Fluent simulation based on the last position (obtained at the end of the unsteady cosimulation) of the PTS3. 


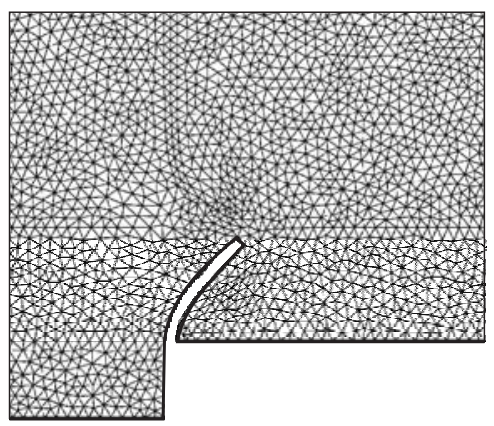

(a)

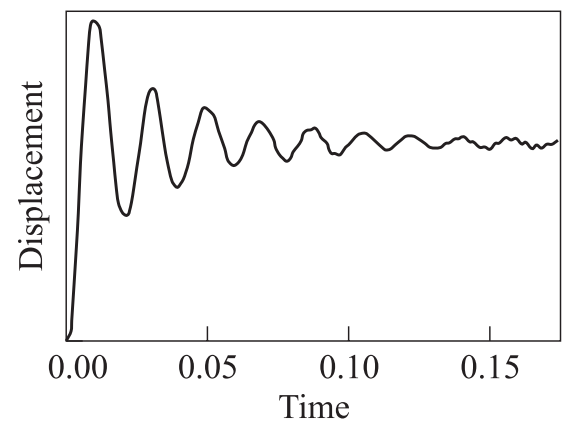

(b)

Figure 14 Maximum bending mesh of the PTS3 (a); and PTS3 tip spatial displacement along the $x$ coordinate $(b)$

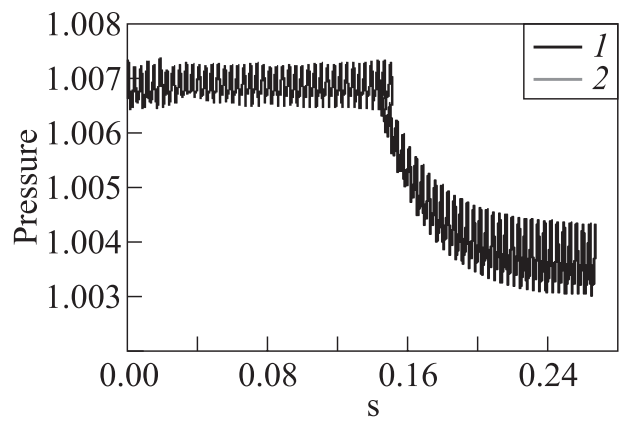

Figure 15 Pressure signal: 1 - static PTS3; and 2-dynamic PTS3

Figure 15 shows that the pressure signal reaches another value which demonstrates the impact of vibrations on the pressure signal.

\section{CONCLUDING REMARKS}

Two weak coupling strategies have been detailed in this paper. The first is oneway coupling, which can be used if the strain of the moving solid surface does not modify the flow field. This strong hypothesis has been made for the test case with solid motor ignition. This simplified approach is useful to quickly obtain first results necessary for a preliminary analysis. Moreover, these results have been obtained by developing a particular script exchanging values and providing a freedom with regard to coupling different commercial software. 
In order to take into account the influence of the propellant surface displacement on the flow field, complementary development was needed. Therefore, the second strategy that is two-way coupling, based one the MpCCI software, has been used for the test case with SRM pressure oscillation phenomena. In the study, the influence of the bending of the PTS3 on the pressure signal as well as the influence of the vibrations was established. Other numerical studies should be performed and completed by experimental campaigns to confirm that the results obtained clearly quantified the real physical phenomena and were not disturbed by numerical artefact. Moreover, the two-way coupling strategy used was still prohibitive in CPU time and other algorithms should be tested.

\section{REFERENCES}

1. Fluent 6.3. User Guide.

2. Abaqus 6.7. User Guide.

3. MpCCI version 3.0.6. User Guide.

4. Geuzaine, C., and J.F. Remacle. Gmsh Reference Manual.

5. Plourde, F. 1994. Structure turbulentes d'un écoulement segmenté à injection pariétale. Thèse de doctorat Université de Poitiers.

6. Couton, D. 1996. Analyse des transferts d'énergie cisaillée par injection pariétale aspect instationnaire. Thèse de doctorat Université de Poitiers.

7. Vetel, J. 2001. Interaction des structures pariétales sur le développement instationnaire d'écoulements cisaillés en milieu confine — rôle de l'injection différentielle. Thèse de doctorat Université de Poitiers.

8. Chi Cong Nguyen. 2002. Effet de la conicité. Interaction des mécanismes tourbillonnaires en espaces confiné. Thése de doctorat Université de Poitiers.

9. Fiedler, R. A., M. S. Breitenfeld, X. Jiao, A. Haselbacher, P. Geubelle, D. Guoy, and M. Brandyberry. 2002. Simulations of slumping propellant and flexing inhibitors in solid rocket motors. AIAA Paper No. 2002-4341.

10. Wasistho, B., R. A. Fiedler, A. Namazifar, and M. D. Brandyberry. 2005. 3D Coupled simulations of flexible inhibitors in the RSRM. AIAA Paper No. 2005-3996.

11. Rey, C., V. Froment, M.-P. Errera, B. Truffart, and A. Langlois. 2007. Numerical investigation of fluid-structure interaction in a model of solid propellant motors. 2nd European Conference for Aerospace Sciences.

12. Projet CPS: code Aérothermochimie propulsion spatiale CPS1.3: Manuel utilisateur/dossier de modélisation. 